\title{
IMPROVED PRE-COPY APPROACH FOR A SECURITY BASED LIVE VIRTUAL MACHINE MIGRATION IN CLOUD COMPUTING
}

\author{
Bindiya \\ Research Scholar, Dept. of CET, \\ Guru Nanak Dev University, Amritsar, Punjab, India \\ Dr. Sandeep Sharma \\ Department of Computer Science Engineering and Technology, \\ Guru Nanak Dev University, Amritsar, Punjab, India
}

\begin{abstract}
In current time Cloud Computing is the most recent pattern where IT applications and foundations are provided as 'administrations' under a use based installment model to its end-clients. Normally two issues happen amid relocation of CPU or memory concentrated VMs which are known as complete movement time and memory utilization at host which results in corruption in the general execution of the framework. In this paper we have proposed a method that removes the memory utilization, utilizing improved pre-copy methodology. So as to expand the security of the data and to limit the information loss, a safe relocation has been accomplished by utilizing the Elgamal cryptographic technique.
\end{abstract}

Keywords: Cloud computing; Virtualization; Live migration; Pre-copy; Post-copy, Security, Elgamal key.

Cite this Article: Bindiya and Dr. Sandeep Sharma, Improved Pre-Copy Approach for a Security Based Live Virtual Machine Migration in Cloud Computing, International Journal of Computer Engineering and Technology, 10(2), 2019, pp. 196204.

http://iaeme.com/Home/issue/IJCET?Volume=10\&Issue=2

\section{INTRODUCTION}

Virtualization is a procedure of using computing assets. Distinctive dimensions of the application framework are separated by it which incorporates programming, equipment, information, systems administration, stockpiling, etc, parts the division among the server farm, stockpiling, servers, information, organizing and the physical gadgets, comprehends dynamic basic structure, and accomplishes the objectives of focal overseeing and powerfully uses the physical just as virtual assets. [1] 
In this paper some serious issues in the information migration in Cloud computing is intended to be settled. The serious issues amid the procedure of information migration are security of the information and information misfortune which is being relocated. Another significant zone of worry in the migration procedure is absolute migration time and memory utilized. So as to take care of the issue of memory starvation these issues are to be tended to and the memory support must be decreased as well. An approval method is being used to give care of the information amid migration.

This paper is sorted out as follow. Area II gives the foundation consider. In area III, a concise review of live migration systems is given. Area IV examines the displaying and design of our proposed work. Segment V talks about the framework execution. Segment VI gives the outcomes. At long last in Section VI, we finish up the paper.

\section{PREVIOUS WORK}

In this part we have examined the few methodologies utilized before. In [3], to decrease the surplus transport of pages a methodology of upgraded pre-copy with characteristic based compression (CBC) calculation is utilized which results in accommodating decrease in migration time and downtime yet utilizing this compression procedure results to information misfortune. To diminish the quantity of transmitted memory amid live migration a strategy [7] called "memory reusing" is utilized.

Anju Mohan et al. sent a manner by which the migration time can be cut somewhere near moving the page that don't appear to be recently used and as opposed to exchanging the grimy pages again the register reports of changes can be sent. Yet at the same time anyway the issue of downtime is still there. In memory investigation and encoding (ME2) approach [9], vital pages are recognized and after that run length encoding (RLE) compression system is used to lessen migration time. Still it isn't dependable as a result of the compression overhead. As depicted by P. Svard et al. [10] execution of the capacity of delta compression all through the vehicle of memory pages to accomplish extend migration throughput and thus limiting the downtime. This calculation for live migration is utilized as an adjustment to the KVM hypervisor. Extra overheads are acquainted due with the compression activities.

To abstain from moving of copy pages and furthermore the moving of free memory pages in [3] post-copy procedure with adaptable pre-paging, dynamic self-swelling process is used so as to improve the absolute migration time and furthermore all out pages being exchanged. In any case, downtime overhead is downside of this methodology. Information replication in virtual machine [5] is a methodology in which the self-comparability of moving memory picture of machine is seen. Based on the above perceptions, this paper presents a way to deal with improve the execution of the live virtual migration and security. So consequently to improve the execution of migration and verification for information security and to diminish the migration time we are utilizing an improved pre-copy approach. In this methodology by diminishing the span of memory support the execution of the live migration improved and for approval Elgamal open key calculation is utilized.

\section{ANALYSIS OF LIVE MIGRATION TECHNIQUES}

The procedure of memory migration can be cultivated by adhering to the means as appeared in figure 1. Pre-copy [2] and Post-copy are two kinds of live migration instruments. [7] VM's memory substance are transported in Post-copy migration process after its processor position has been sent to the pointed host. Not at all like Post-copy approach in pre-copy approach, initially the duplicates of memory state are sent to the goal, all through a repetitive procedure, 
after which its processor state is transported to the item .The beneath referenced stages are kept Pre-copy migration [5]:

- In first emphasis, while the whole memory pages are duplicated to the goal VM, the source VM keeps working. Just those pages that were adjusted amid the before exchange round will reproduce in progressive emphases.

- Then the source VM is shut; the CPU state and remaining grimy pages are imitated. The goal VM is started.

- The most recent VM starts to work; on the off chance that it endeavors to get to a page that has not so far been exchanged; from the source VM this page has been acquired.

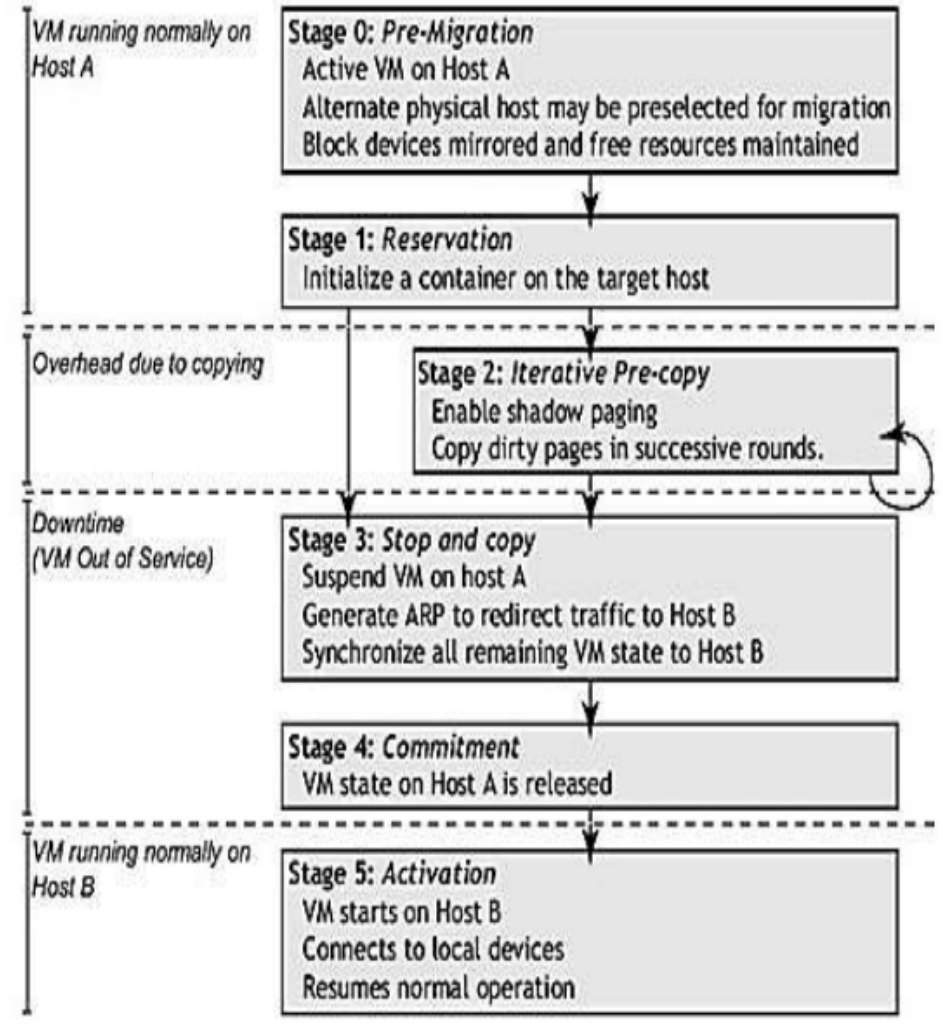

Figure 1. Migration process [2]

Post-copy migration [10] basically pursues the following stages:

- VM is suspended at the source have. The condition of the processor is sent to target VM, and the conditions of gadgets are imitated with the substance of virtual CPU registers are imitated at the goal.

- Recommence the VM at the objective select of any memory content.

- The VM is incidentally halted and furthermore the defective pages are request paged over the system from the source regardless if the VM endeavors to get to pages that have not so far been exchanged. The VM is begun once more.

\section{PLANNING \& DESIGN}

With the learning of all out migration time and memory utilization and information exchange rate execution of the virtual machine migration can be determined. [10] The memory picture of VM is continued in the source have when a virtual machine movements to another host, which is clarified prior by various creators. 


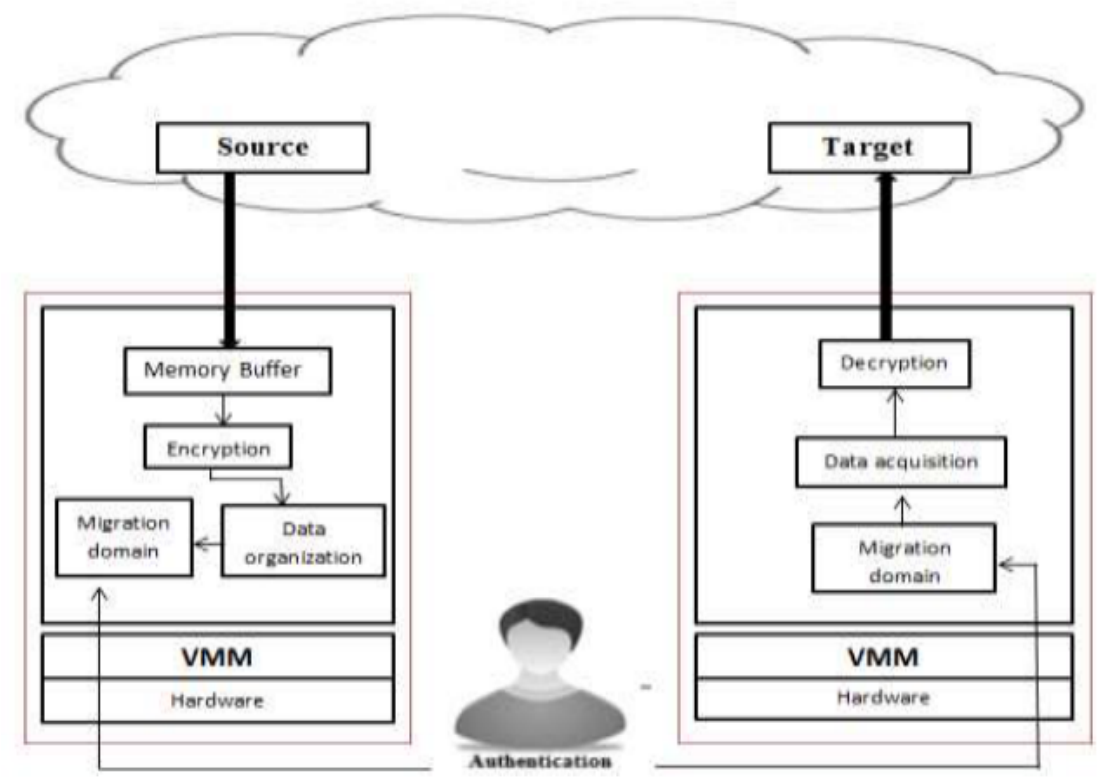

Figure 2. Pre-copy based encryption technique

The held memory picture will be "reused" right when the VM is migrated to the first host by and by a short time later. So as to improve VM positions a few VM migration procedures are used. [7] For any situation, these procedures tend to prompt extensive system traffic, as open migration frameworks switches the entire memory picture of the objective VM. This prompts delay during the time spent migration and defers the accomplishment of the VM positions improvement.

In the previous methodology amid the information migration, information is compressed and after that compressed information is exchanged utilizing hashing calculation for security while exchanging starting with one host then onto the next in live migration. Compressing the information will prompt some twist in the data. Amid the memory exchange, we need to concentrate on decreasing the information sum exchange volume and the memory that is put away in the host for time.

Pre-copy is done as an essential copy of the entire memory page pursued by the rehashed duplicates of the altered pages all through the prior copy from the source host to the goal or focus until the quantity of changed pages is similarly little. [10] As delineated in fig.2, the information from source have is scrambled and cushioned by the information association module and sent to the objective hub. In the objective hub, the information procurement module initially unscrambles the information and after that further preparing happens. The migration forms are prohibited underneath the migration space. Amid the pre-copy stage, the alteration to the memory page is returned to the source machine.

Access the board is a crucial angle for security in this proposed strategy, Elgamal calculation is clarified and set in motion for the capacity of the ensured approval amid VM migration. The calculation is based on deffie hallman key swap calculation which comprises of topsy-turvy key encryption. After the critical information is put away on cloud, the information is then encoded by an open key utilizing Elgamal calculation. By utilizing private key the scrambled information is unscrambled. The advantage of Elgamal encryption calculation is that, on the off chance that there is same plain content, at that point it assigns extraordinary figure message each time it is scrambled making it increasingly ensured against reversal assault. To secure information while moving in VM, Elgamal calculation is used. 


\section{METHOD UTILIZATION}

Cloudsim 3.0.3 coordinated with Java Runtime Environment in Net-beans IDE 7.4. Java is utilized to structure the interface for VM migration to supply information though for handling to the VMs, MySQL is utilized. Information is put away in type of tables. At the point when a VM is instated, it gets the qualities from the table. In MySQL an each different table is put away with number of fields in each VM. In this area we portray the distinctive structure strategies, through which we can compute the outcomes.

The well-ordered strategy of the proposed secure VM migration utilizing improved precopy approach and verification

Pseudo code for improved pre-copy
approach

1) Initialize the CloudSim library

2) CloudSim.init(num_user, calendar, trace_flag);

3) Create host and datacenters using constructors

Host $h=$ new Host (hostld, new
RamProvisionerSimple(ram),
New BwProvisionerSimple (bw),
storage, peList2, new
VmSchedulerTimeShared (peList2))
Data Center datacenter = new
Datacenter
(name, characteristics, new
VmAllocationPolicySimple
(hostList), storageList, 0);

4) Create virtual machines and cloudlets and initialize $\mathrm{vm}$ with data from database

$V m v m I=$ new NewVm (vmid, brokerId, mips, pesNumber, ram, bw, size, vmm, new CloudletSchedulerTimeShared()); Cloudlet cloudlet $1=$ new Cloudlet (id, length, pesNumber, fileSize, outputSize, utilizationModel, utilizationModel, utilizationModel);

5. Initialize Host $A$ host. initiailize 0;

6. Calculate migrating memory host.getmigratingmemory $O$

7. Move from host $A$ to host $B$ moveAtoB O

8. Perform updation on memory pages updatepages 0

9. Compute changes and calculate memory host. MigratingMemoryHostB 0 ;

10. Received changes and migration completed.

technology is depicted in figure 3. So as to limit the memory use, entire information isn't transported starting with one host then onto the next and just the information which is required will be introduced and thus just that information will be migrated. At whatever point a VM is prepared for relocation, its memory picture of the state is recorded in the host, with the goal that it tends to be utilized in future when information of that VM is exchanged once more from the goal host to that have again so as to diminish the memory utilization. This memory picture is recorded in that have until the movement procedure finishes and consequently when the quantity of VMs are extensive then the huge measure of memory piece is used which will prompt memory utilization emergency. Because of this, host won't most likely instate other virtual machines and furthermore won't probably acknowledge any VM's information amid movement. 


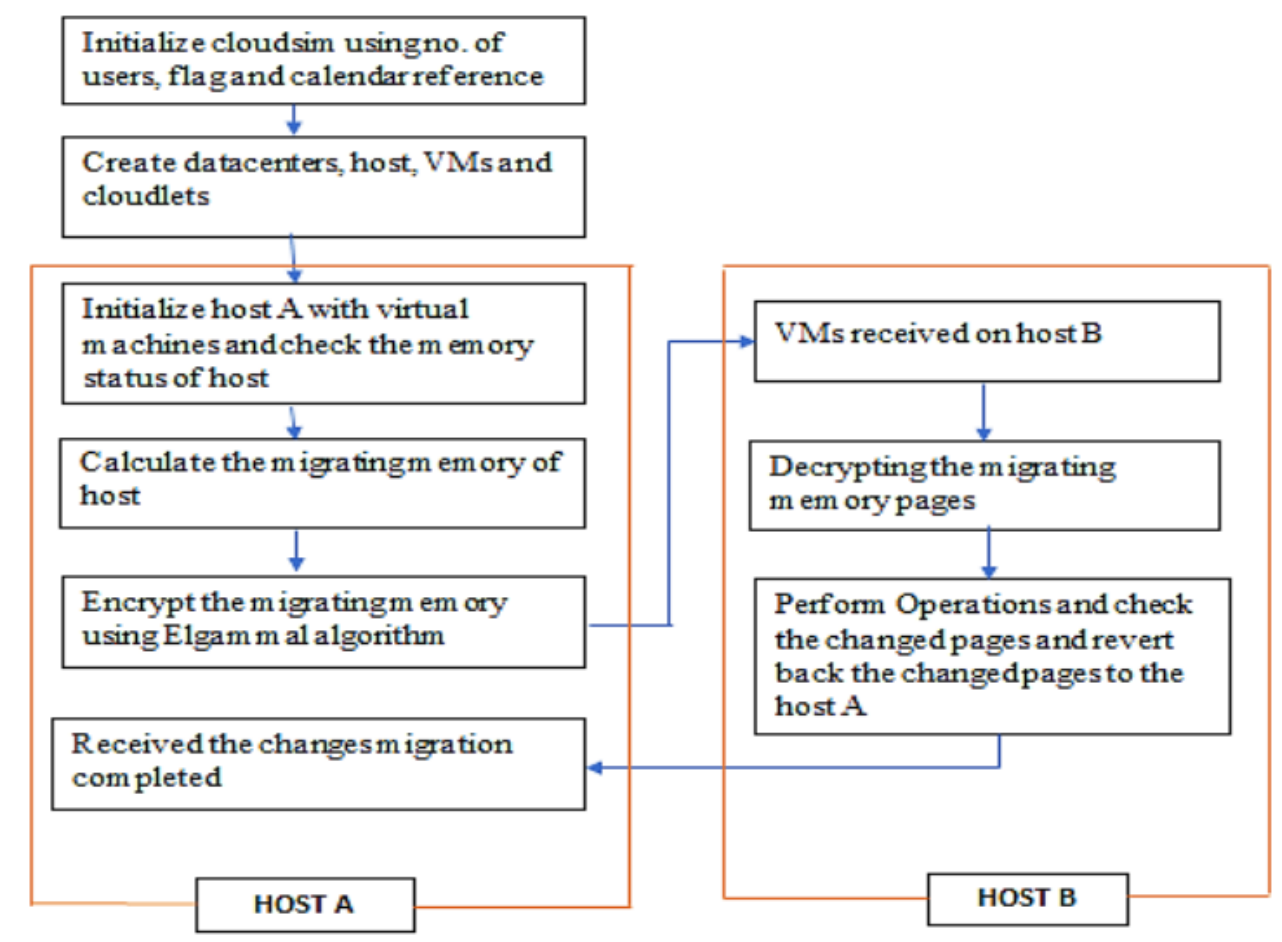

Figure 3. Flow chart of proposed approach

In our proposed structure we will endeavor to defeat this issue by introducing and exchanging just those memory pages that are required. The memory page that doesn't have the likelihood of refreshing on another host need not be instated and sent. Thus, the memory pages which change as often as possible will won't to be stored in memory. By applying these two systems, memory utilization amid the movement in pre-copy approach can be diminished to some degree.

To diminish the information loss because of assault on memory space and to build the secrecy of the information which is being moved, a verified relocation is finished by utilizing the cryptographic calculations. In this, Elgamal open key cryptographic calculation is used to encode the memory pages which must be relocated starting with one host then onto the next host.

\section{OUTCOMES}

This section presents the results and discussion of live VM migration experiments and addresses the three main performance metrics; total migration time, memory consumption and encryption time.

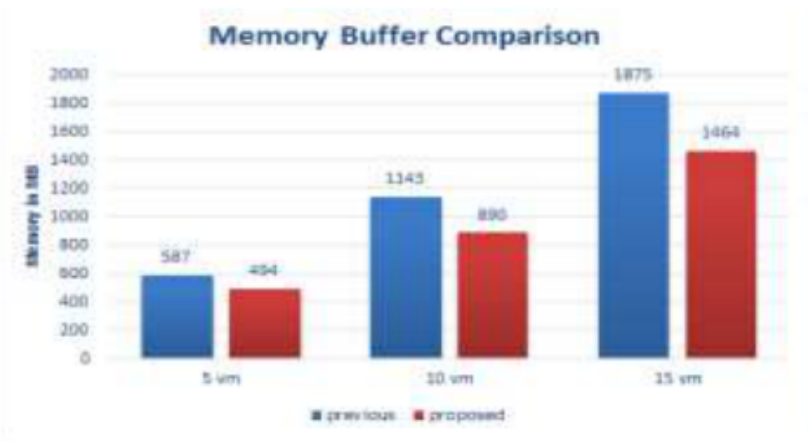

Figure 4 Memory buffer comparison 
Diverse situations are utilized for example $5 \mathrm{VMs}, 10 \mathrm{VMs}$ and $15 \mathrm{VMs}$ are utilized. For every situation, we compute size of memory cushion at source have, all out relocation time and encryption time.

\subsection{Reduction in memory utilization of information}

In our proposed framework we diminish the measure of the memory cradle as talked about in area $\mathrm{V}$, that is memory starvation, which has been settled by applying the accompanying conditions, I) information that has less or zero likelihood of update on another host and ii) information having incredible likelihood of refreshing of progress in a not so distant future shouldn't be incorporated. The distinctive sort of outstanding task at hand utilized as appeared table I.

Table I: Type of workload

\begin{tabular}{|c|c|l|}
\hline & Name & \multicolumn{1}{|c|}{ Description } \\
\hline 1 & Busy loop & CPU: Infinite busy loop \\
\hline 2 & Apache & Network, Input/output \\
\hline 3 & Video & Input/output \\
\hline 4 & TPC-C & $\begin{array}{l}\text { Databases which simulates } \\
\text { transactions of an online shop }\end{array}$ \\
\hline
\end{tabular}

Examination between both the strategies demonstrates that, the proposed framework is smarter to utilize memory in proficient manner as it can utilize increasingly number of virtual machine by utilizing less memory when contrasted with existing procedure as appeared in figure 4 .

We figure the decrease proportion as indicated by the underneath condition which characterizes, how effective is the proposed framework.

$$
\begin{array}{r}
\mathbf{R}=\mathbf{M} \text { previous }-\mathbf{M} \text { proposed } / \mathbf{M} \text { previous } \\
=1875-1464 / 1875 \\
=22 \%(\text { approx...) }
\end{array}
$$

Where, $\mathrm{R}$ represents decrease in size of memory buffer, Mprevious represents memory size of information for existing strategy and $M$ proposed for proposed system. In this manner, the outcome demonstrates that proposed methodology limit the memory buffer estimate contained on the source have by $22 \%$.

\subsection{Comparison with existing framework}

Similarly, we are getting the aftereffects of existing procedure by actualizing them in a similar situation. It demonstrates that calculation incredibly improves the execution of live movement. As found in figure 5, relocation time is improved by $9 \%$ by utilizing a similar decrease condition. Likewise, the real concern while moving virtual machines from source machine to goal machine is their security. Elgamal cryptographic method is utilized to give high security as appeared in figure 6. More the encryption time more will be the security which give secure confirmation and classification while VM relocation. 


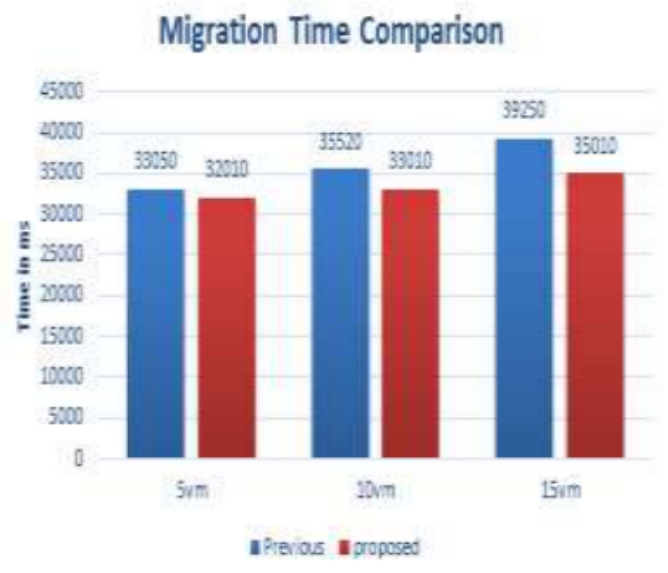

Figure 5 Migration time comparison

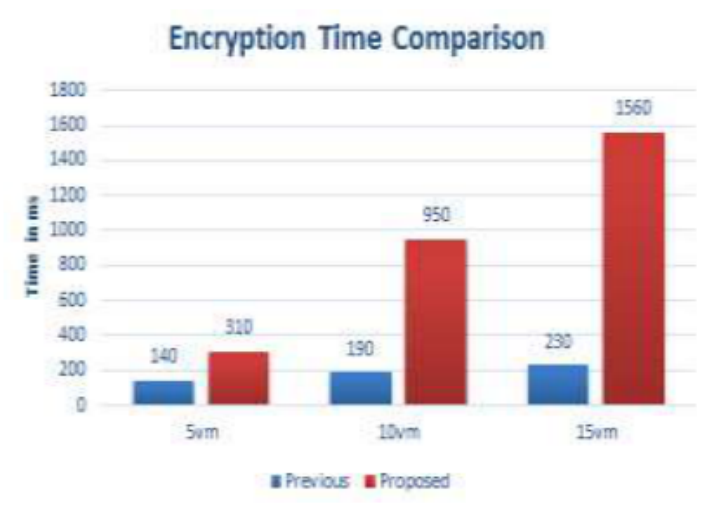

Figure 6. Encryption time comparison

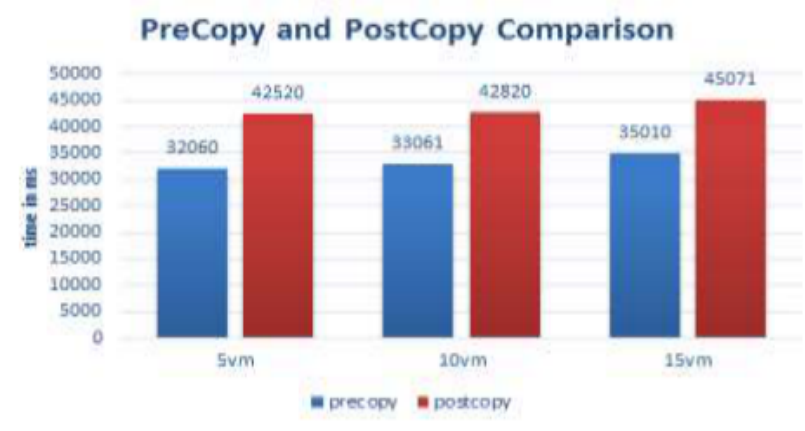

Figure 7 Pre-Copy and post copy comparison

Figure 7 demonstrates the complete movement time correlation of two methodologies. In this way from the outcomes we infer that the general execution of Pre-copy is much prevalent than the post-copy and it demonstrates that the execution measurements are improved of all relocation calculations.

\section{CONCLUSION}

In virtualization, live virtual machine relocation is one among the deepest trademark. In this paper, we have proposed an imaginative system to move information on cloud utilizing improved pre-copy approach. We have basically centered around capable memory managing for source have which will likewise limit the necessities of memory amid relocation. By doing this we can utilize increasingly number of virtual machines with less memory prerequisites. Execution metric memory utilization and relocation time decreases at an extraordinary degree. The confirmation plot diminishes the loss of information amid the movement and furthermore furnishes with secure relocation. In future the framework can be made increasingly exact, with beneficial change.

\section{REFERENCES}

[1] S. Zhang, H. Yan, X. Chen,” Key Technologies of Cloud Computing” In International Conference on Medical Physics and Biomedical Engineering, 33 (2012) 1791 - 1797.

[2] Santosh Kumar Majhi, Sunil Kumar Dhal "A Security Context Migration Framework for Virtual Machine Migration" IEEE Intl. Conference on Computing and Network Communications (CoCoNet'15), Dec. 16-19, 2015 
[3] Megha R. Desai” Efficient Virtual Machine Migration in Cloud Computing” IEEE Fifth International Conference on Communication Systems and Network Technologies, 2015.

[4] Sagana C, Geetha M, Suganthe R. Performance enhancement in live migration, International Conference on Information Communication and Embedded Systems (ICICES); 2013.

[5] Lin C-C, Huang Y-C, Jian Z-D. A two-phase iterative pre-copy strategy for live migration of virtual machines, ICCM, IEEE; 2012

[6] S. Akiyama, T. Hirofuchi ," MiyakoDori: A Memory Reusing Mechanism for Dynamic VM Consolidation" IEEE Fifth International Conference on Cloud Computing, 2012

[7] Anju Mohan, Shine S," An Optimized Approach for Live VM Migration using Log Records" IEEE 2013

[8] Y. Ma, H. Wang, J. Dong, Y. Li, and S. Cheng, "ME2: efficient live migration of virtual machine with memory exploration and encoding", IEEE International Conference on Cluster Computing, pp 610-613, 2012

[9] P. Svard, J. Tordsson, B. Hudzia, and E. Elmroth 2011. "High Performance Live migration through dynamic page transfer reordering and compression" 3rd IEEE International Conference on Cloud Computing Technology and Science, Cloud-Com, 542548.

[10] T. Hirofuchi, H. Nakada, S. Itoh, and S. Sekiguchi. "Reactive consolidation of virtual machines enabled by post copy live migration", In Proceedings of the 5th international work-shop on Virtualization technologies in distributed computing, VTDC '11, pages 1118, New York, NY, USA, 2011. ACM. 